\title{
Solar activity and its influence on climate
}

\section{C. de Jager}

Royal Netherlands Institute for Sea Research, P.0. Box 59, 1790 AB Texel, the Netherlands. Email: cdej@kpnplanet.nl

Manuscript received: June 2008; accepted: September 2008

\begin{abstract}
Solar activity, as manifested by its many equatorial as well as high-latitude components of short-term variability is regulated by the Sun's dynamo. This constitutes an intricate interplay between the solar toroidal and poloidal magnetic field components. The dynamo originates in the tachocline, which is a thin layer situated about $200,000 \mathrm{~km}$ beneath the solar surface. The dynamo is a non-linear system with deterministic chaotic elements, hence in principle unpredictable. Yet there are regularities in the past behaviour, such as the Grand Maxima (example: the recent high maximum of the 2nd half of the 20th century) the Grand Minima (e.g. the Maunder Minimum between 1650 and 1710) and the Regular 0scillations such as those between 1730 and 1923. Their occurrences are described by a phase diagram in which a specific point can be identified: the Transition Point. This diagram plays an essential role in determining the future solar activity. Guided by its quasi-regularities and by recent measurements of the solar magnetic fields we find that the Sun is presently undergoing a transition between the past Grand Maximum and a forthcoming period of Regular Oscillations. We forecast that this latter period will start in a few years and will continue for at least one Gleissberg cycle and that the next solar maximum (expected for 2014) will be low $\left(R_{\max } \sim 68\right)$.

We discuss the heliospheric drivers of Sun-climate interaction and find that the low-latitude magnetic regions contribute most to tropospheric temperatures but that also the influence of the - so far always neglected - polar activity is significant. Subtraction of these components from the observed temperatures of the past 400 years shows a residual series of relative peaks and dips in the temperature. These tops and lows last for periods of the order of the Gleissberg cycle. One of these is the recent period of global warming, which, from this point of view, is not an exceptional period.
\end{abstract}

\section{Equatorial and polar manifestations of solar activity}

The Sun is a gaseous globe with an effective temperature of about $5800 \mathrm{~K}$. It is surrounded by a hot, tenuous and fairly irregular cloud of plasma with a temperature of a few million degrees: the solar corona. The corona can only be seen visually during total eclipses.

The Sun owes its energy to internal hydrogen-helium fusion reactions, and it is a very stable radiator. The radiated energy flux increases slowly and steadily with time, by slightly less than $1 \%$ per 100 million years.

Apart from this very constant behaviour there is a variable constituent, with many components that are for one part situated in the near-equatorial regions, up to latitudes of some $35^{\circ}$ to $40^{\circ}$, while other manifestations of solar activity are situated at higher latitudes. In this paper these two components of solar magnetic activity, the high-latitude and the equatorial one, are described. We will show that they are related, in a delicate way, to the poloidal and toroidal components of a deeper seated magnetic field. These two components alternate in structure and magnetic fieldstrength in the course of time. We will show in this paper that their variation is related to the Earth's tropospheric temperatures. Below we first summarize the visible solar structures (cf. review by De Jager, 2005). Thereafter we discuss their origin in the solar dynamo.

\section{Equatorial features}

Very visible manifestations of the Sun's variable activity are the sunspots. These are regions with very varying diameters. They may be as large as the Earth, which is some $12,000 \mathrm{~km}$ 
and sometimes their diameter can be as much as $25,000 \mathrm{~km}$. Their main feature is the occurrence of strong vertical magnetic fields, with fieldstrengths up to some 3500 Gauss (i.e. 7000 times the average strength of the magnetic field at the Earth's surface). These strong magnetic fields inhibit the convective motions that transport energy upward; consequently the sunspots are relatively dark. Yet their effective temperature is about $4500 \mathrm{~K}$; hence their darkness is only relative with regard to the surrounding solar photosphere.

The number of sunspots visible on the solar disk has been counted continuously since 1610 and provides a simple measure of solar activity. Characteristic is the Schwabe cycle during which the spots' magnetic fields feature changes in a 11 year period (Fig. 1). The long term amplitude modulation of the sunspot cycle that appears to regulate most aspects of solar variability is composed by the Gleissberg cycle. This is one of the periods occurring in the time-variation of solar variability. Its value is variable over the centuries, ranging between 60 and 140 years with an average value of 88 years (Ogurtsov et al., 2002). Another component affecting the long-term variation of the Sun is the De Vries or Suess cycle. This is a fairly constant period of variability that peaks fairly sharply at about 205 years. The Hallstatt cycle of 2300 years also seems to be of solar origin (Clilverd et. al., 2003; Muscheler et al., 2003). 0ther cycles sometimes attributed to the Sun (Stoykova et al., 2008) may be ascribed to atmospheric periodicities.

The magnetic fields of sunspots show strict regularities (the polarity laws) that change in a 22 years cycle. This makes clear that the essential physical period of the Sun is not the 11-years Schwabe cycle but a cycle of twice its duration: the 22-years Hale cycle.

The sunspots are the core features of the active regions. The latter are regions, typically of sizes of some $200,000 \mathrm{~km}$ (in longitude) by 50,000 km (latitude) in size, which surround the sunspots (Fig. 2). We will show below that the magnetic fields of the active regions originate in deeper layers and are convectively brought to the solar surface. The main feature of the active regions is the large number of magnetic flux tubes. Each of these tubes is confined magnetically and carries electric currents of $10^{9}$ ampères or even more. Dissipation of their magnetic energy causes locally heated areas in the active regions, the so-called faculae, that have temperatures around $10,000 \mathrm{~K}$, in contrast to the undisturbed parts of the solar surface which have lower temperatures. Because of their higher temperatures the faculae are sources of relatively strong UV radiation. The active regions vary in strength and size in the course of the eleven years solar cycle and therefore the UV radiation flux also varies strongly, in parallel to these variations. Since the higher temperature of the faculae is indirectly (through dissipation processes in magnetic flux tubes) related to the general magnetic field of the active region, these magnetic fields are relevant for Sun-climate relationships.

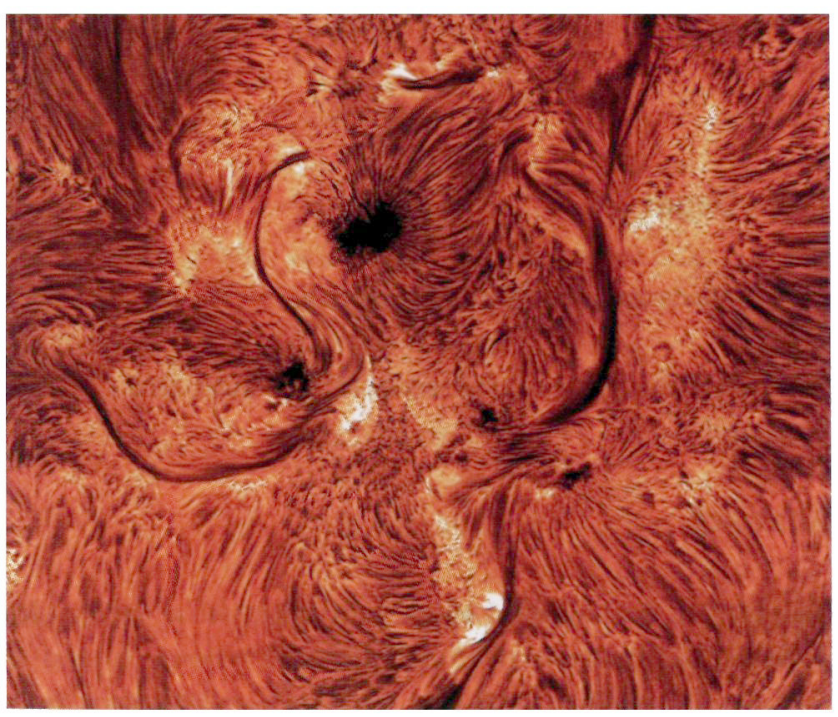

Fig. 2. An active region: note the numerous magnetized current tubes around sunspots.

Close to the sunspots, flux tubes with much stronger electric currents are present. Currents of up to $10^{12}$ amp may occur. Coalescence of such flux tubes (review by Sakai \& de Jager, 1996) can result in solar flares: short lived explosive phenomena. Their impulsive phase lasts on average for a few to some tens of seconds, and only exceptionally much longer. During that phase temperatures of the order of 50 million degrees and higher are reached (De Jager, 1983). In one case temperatures of 400 to 500 million $\mathrm{K}$ were observed in 13 subsequent pulses, each lasting for about $0.1 \mathrm{~s}$ (De Jager et al., 1987). The average energy of a solar flare is comparable to that of $10^{9}$ Hirojima bombs. Occasionally a solar flare results in a flare surge, which is a 'tongue' of hot plasma, rising with velocities of a few tens of $\mathrm{km} / \mathrm{sec}$, up to heights of 50,000 to $100,000 \mathrm{~km}$ above the solar surface. Their lifetimes range between 5 and 60 minutes.

Another feature, related to, but not caused by solar flares is the Coronal Mass Ejections ('CME'; review by Hundhausen, 1999). In a Coronal Mass Ejection plasma is emitted from the Active Centre, sometimes over a large region of the solar surface that may span longitudinal intervals of more than $90^{\circ}$. The
Fig. 1. Sunspot numbers since 1610, showing the 11-years cycle.

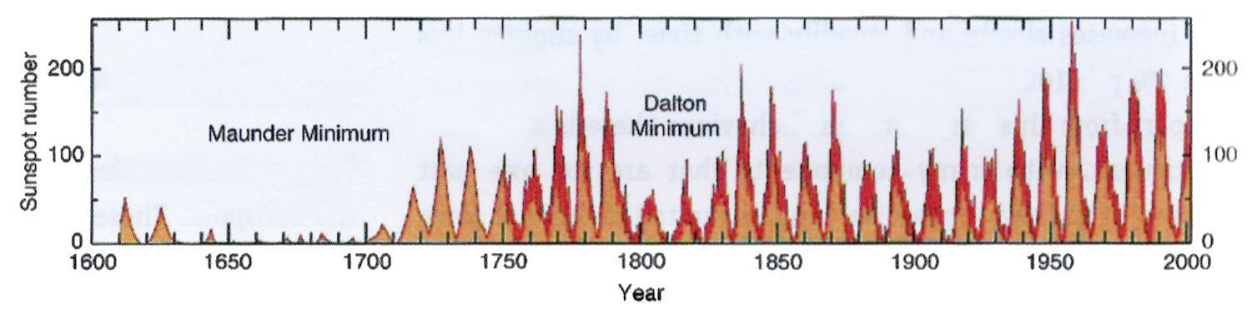


plasma leaves the Sun with velocities of the order of a few tens of $\mathrm{km} / \mathrm{s}$ but it is accelerated underway till, in the vicinity of the Earth's orbit, velocities of the order of 200 to $2500 \mathrm{~km} / \mathrm{s}$ (with an average of $400 \mathrm{~km} / \mathrm{s}$ ) are reached. The CMEs carry magnetic fields along that tend to fill the heliosphere - the magnetically closed region around the solar system. These interplanetary magnetic fields only slowly diffuse away. Diffusion times of the order of some 4 years are common. The CMEs may appear to be important for better understanding the Sun-Earth relations, because they change the magnetic contents of the heliosphere in an important way, while this happens during fairly long periods of time because of the slow diffusion.

Rather unlike these very dynamic solar features are the filaments and the prominences (= a filament seen at the Sun's limb). These are rather stable structures, though with striking internal motions. They tend to last for days to weeks, sometimes even longer. They are commonly situated along the magnetic neutral line at the surface of an active region. They differ from the short lived flare filaments that occur in flaring regions, and which have short lifetimes and are related to the flare surges.

The active regions are also the seats of the coronal streamers. These are the long structures that were initially only seen during solar eclipses, extending from the Sun to distances of many solar radii. Nowadays they are also observed with coronagraphs in space.

\section{Polar features}

The polar regions of the Sun contain various kinds of phenomena. Polar faculae are small-scale emission features (Erofeev and Erofeeva, 2000). They occur at latitudes above $40^{\circ}$, on average at latitude $55^{\circ}$, and have weak magnetic fields. Their magnetic field strength is typically of the order of a few to several tens, exceptionally up to a hundred, Gauss. Yet, the polar faculae are so common that their integrated magnetic flux is a few times larger than that of the equatorial faculae. However, this does not hold for their total magnetic energy, because that is proportional to the square of the fieldstrength (Callebaut, pers. comm.).

The polar bright points are similar structures but with much stronger fields.

Very striking are the coronal holes (Fig. 3), which are extensive areas where the solar surface, as observed in X-rays is relatively dark. This is due to the fact that at their location the corona has a low temperature and a low density. Coronal holes have weak or no magnetic fields and are sources of the solar wind, as they emanate plasma streams (Shrivastava \& Jaiswal, 2003). Of these plasma streams, the high-speed ones may appear to be important for the Sun-Earth connection, because they carry magnetic fields into interplanetary space (Richardson, 2004; see also Akasofu \& Frey, 1986). These fields may surround the Earth and thus influence the terrestrial magnetic field with possible consequences for the Earth's climate.
Many coronal holes are situated in the polar regions of the Sun, but some of them may extend down to and sometimes even across the equator (cf. Fig. 3).

Also contributing to the transport of plasma from the Sun towards the Earth are the ephemeral active regions (Harvey \& Martin, 1973; Lockwood et al., 1999). Ephemeral active regions appear mostly as small bipolar regions. Typically, they have sizes of the order of about $30,000 \mathrm{~km}$. Per day up to some hundred ephemeral active regions may originate. The total amount of magnetic flux in these regions is impressive. It is comparable to that of large active regions. When ephemeral active regions come and go, magnetic fields are carried into space, with possible consequences for the terrestrial environment.

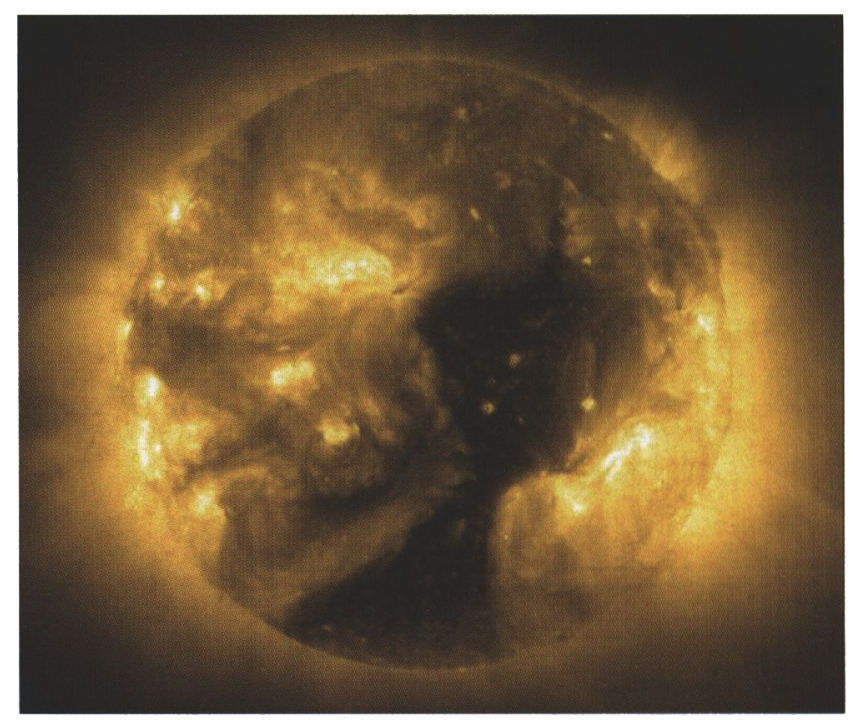

Fig. 3. A coronal hole.

\section{The solar dynamo}

The features described above are driven by the solar dynamo (Spiegel \& Weiss, 1980, Brun, 2004, Nandy, 2004, Dikpati et al., 2004). This solar machine is seated in the tachocline, which is a relatively thin ( $40,000 \mathrm{~km}$ thick) layer at a depth of about $200,000 \mathrm{~km}$ below the Sun's surface. At that level, at the bottom of the Sun's convective region, magnetic fields are amplified by shearing motions caused by interaction between the convective motions and the solar rotation, in combination with an inner weak poloidal field. The Sun's differential rotation, with the shortest rotation period at the equator, causes magnetic fields that are initially poloidal to become stretched in a direction parallel to the equator. This causes them to change their orientation into a toroidal field. By this action the magnetic fields are amplified. This can be easily visualized: the field lines become more closely spaced. This is the Omega effect.

The equilibrium fieldstrength at the tachocline is $10^{4}$ Gauss. When, by continuous further amplification, fieldstrengths of the order of some $10^{5}$ Gauss are reached, the ongoing stretching of the toroidal magnetic loops will result in the detachment of 
parts of the basic magnetic loop. After detachment, the released part of the magnetic flux tube as well as the source flux tube close immediately, because an open flux tube cannot exist. The detached closed loop-shaped flux tube subsequently rises because its specific weight is less than that of the surroundings. Hence it ascends through the outer parts of the Sun, thereby covering a distance of some $200,000 \mathrm{~km}$. The rise time is a few months. When arriving at the surface these magnetic loops manifest themselves as a group of two oppositely polarized sunspots. An opposite polarity distribution of sunspots occurs at the opposite (north vs. south) solar hemisphere.

Weaker magnetic fields may also detach themselves from the toroidal field. They rise more slowly and under the influence of the Coriolis force their upward motion is mainly along the rotation axis of the Sun. At the same time they rotate over about $90^{\circ}$ and so they appear at the surface as mainly poloidally directed fields. This is the alpha effect. This latter process occurs mainly in the periods between maximum sunspot activities, hence between the times when the toroidal magnetic field strengths reach their maximum values. The occurrence of the polar magnetic fields starts in the declining phase of the toroidal field to reach its maximum strength during the sunspot minimum. In other words: maximum strengths of these two magnetic phenomena, poloidal and toroidal, are out of phase by half a Schwabe cycle, hence of the order of five to seven years, because Schwabe cycles may last for nine to fourteen years.

The steady interaction between the poloidal and the toroidal magnetic fields is the main feature of the solar dynamo.

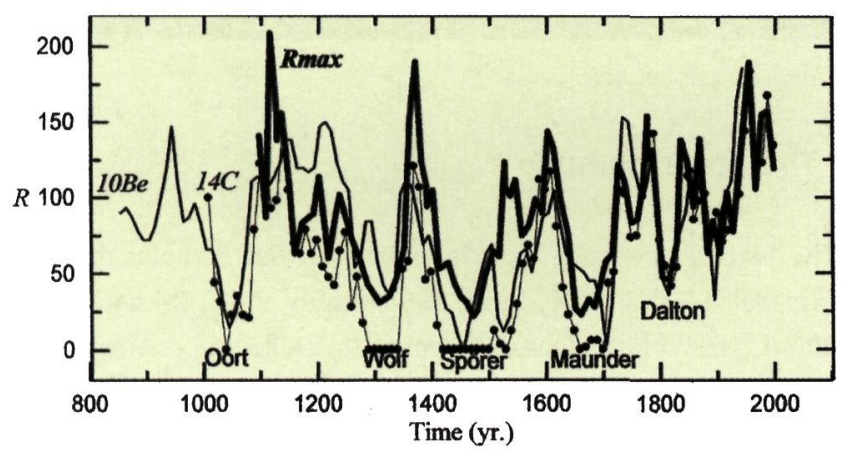

Fig. 4. Smoothed maximum sunspot numbers; a proxy for the maximum toroidal field strength over the centuries (Duhau \& De Jager, 2008).

Hence the maximum number of sunspots, $R_{\max }$, is a proxy for the toroidal field strength (Fig. 4). During the subsequent sunspot cycle the polarities of leading and following spots, as well as those of sunspots on either hemisphere have changed into their opposite signs: the polarity laws. Hence it is physically more correct to speak of the 22-years cycle, the Hale cycle. As is the case with the Schwabe cycle the Hale cycle too is not a strictly periodic process: there are long and short cycles as well as strong and weak cycles.

It is also important to note that that the first spots of a 'new' sunspot cycle already appear, at high latitudes, when spots of the 'old' cycle are still present at low latitudes on the disc: there is overlap between cycles. Sunspots that belong to subsequent cycles are differentiated according to their polarities. For example: the first spot of cycle \#24 appeared at high latitude in the course of December 2007 while in the months thereafter some fairly strong low latitude spots appeared that still belonged to the last cycle, \#23.

A proxy for the poloidal field is $a a_{\text {min }}$, the value of the geomagnetic fieldstrength aa at sunspot minimum (Fig. 5).

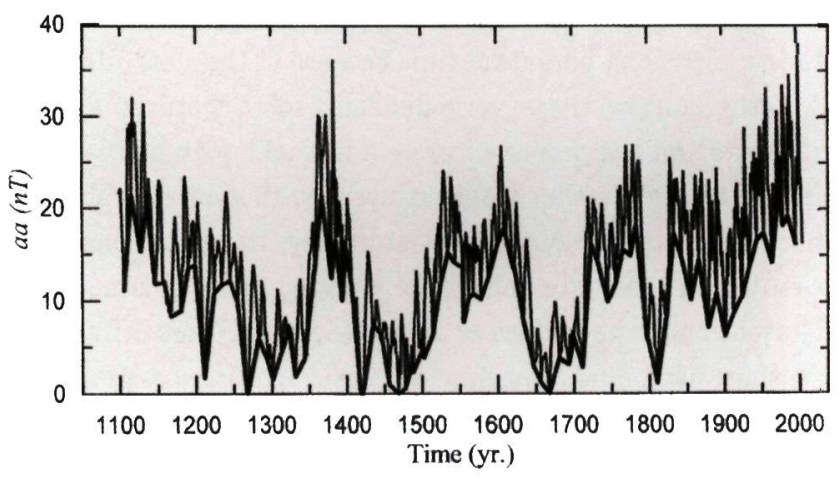

Fig. 5. Smoothed values of the geomagnetic a index at sunspot minimum; a proxy for the maximum poloidal fieldstrength (Duhau \& De Jager, 2008).

\section{Grand Episodes and the forecast of the next cycle (\#24)}

The solar dynamo is a non-linear system with a deterministic chaotic character (Ostryakov \& Usoskin, 1990; Feynman \& Gabriel, 1998; Weiss, 2002; Weiss and Tobias, 2000; Duhau, 2003). Hence it is unpredictable in principle (Tobias et al., 2006). Yet quite a few authors have published forecasts for the timing and magnitude of the maximum strength of the forthcoming solar cycle, \#24 (summarized in De Jager \& Duhau, 2008). In virtually all of these investigations the chaotic character of the solar dynamo has not been taken into account. A way to overcome this modelling issue is to consider the quasi-regularity in the Sun's Grand Episodes. These Grand Episodes are both a reflection of the solar dynamo's chaotic aspect, as well as indicating some regularity that may be helpful in forecasting solar activity.

Figures 1, 4 and 5 show the occurrence of episodes during which solar activity was either exceptionally strong or, conversely, exceptionally weak. We call these Grand Maxima and Grand Minima. An example of the latter is the Maunder Minimum of 1650 - 1710, whereas an example of the former is the Grand Maximum that occurred in the second half of the 20th century. It appears, though, that in addition to these Grand Episodes there is another kind of solar activity feature: the Regular Oscillations. An example of these is the period 1730 - 1923. During that period the proxy values of solar activity oscillated quasi-regularly around an average value, as is shown in Figs 4 and 5. 
A useful way to visualize the various Episodes and their relationships is to plot the proxies of the poloidal fieldstrength $B_{p o l}$ against those of the toroidal one, $B_{\text {tor }}$. Thus one obtains a phase diagram (Duhau \& Chen, 2002; Duhau, 2003, Duhau \& De Jager, 2008). Such a diagram is shown in Fig. 6, where we have plotted the smoothed proxy values in order to eliminate the 11-years cyclic fluctuations. It then appears that there exists a sharply defined point in the diagram, that Duhau \& De Jager (2008) call the 'Transition Point'. It has the coordinates $R_{\text {max }}=93.38 \pm 0.69$ and $a a_{\min }=10.39 \pm 0.08$. At quasi-regular times the $\left(R_{\max }, a a_{\min }\right)$ curve crosses either through or in other cases is passes very close to this point. Each time when the curve crosses precisely through the Transition Point a Grand Maximum or Grand Minimum episode starts. After crossing or passing the Transition Point the course of the curve's motion changes from clockwise to anti-clockwise or vice-versa. In cases that it just 'misses' the Transition Point an episode of Regular 0scillations starts. We show this in the example of Fig. 6, which refers to the period 1730 - present. In 1730 the Maunder Minimum terminated but at that time the curve just missed the Transition Point and thereafter it spiralled, in a counterclockwise motion, around the Transition Point. This is the period of Regular 0scillations that lasted from 1730 to 1923. In 1923 the curve did hit the Transition Point where after the direction of motion became clockwise again. At that time the recent Grand Maximum, that ended a few years ago, started. In 2006 the curve did not cross the Transition Point but passed close to it and this indicates that another period of Regular Oscillations is imminent. Guided by the observations of earlier similar events we foresee that this coming episode will last for one or two Gleissberg cycles, hence for about 50 to 200 years.

Fig. 6. Phase diagram of the long-period components of solar variability for the period 1730 to 2006.

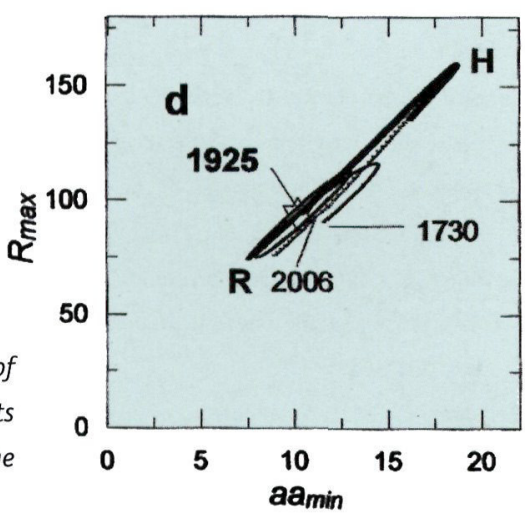

These results have clear implications for our understanding of the solar dynamo. This non-linear system apparently obeys to certain relationships in which a chaotic element is discernable - a tough problem for those solar theorists that are attempting to develop predictive models. Yet, these results do already allow one to forecast future solar activity.

These analysis, together with observations of the polar magnetic fieldstrength during the past few years, in combination with considerations of the long-term and short- term components of solar variability enables us to forecast the maximum strength and time of maximum of the next solar cycle, \#24 (De Jager and Duhau, 2008). We predict a maximum sunspot number of $68 \pm 17$ in 2014 .

\section{Sun-climate relationships}

Terrestrial tropospheric temperatures $T_{\text {trop }}$ gradually and slowly increased since the Maunder Minimum, though with large oscillations - cf. Fig 7 (after Moberg et al., 2005). The same applies to the annual number of sunspots (= solar sunspot number R) (Fig. 8) as well as to the $a a_{\min }$ values (Fig. 9). In a previous investigation we (De Jager \& Usoskin, 2006) found a well-defined linear relationship between $T_{\text {trop }}$ and $R$, but this can be further refined. Previously, the influence of the Sun's polar magnetic activity has always been neglected in Sunclimate studies. At this time we go a step further and ask ourselves what fractions of the equatorial and polar magnetic fields do contribute to $T_{\text {trop }}$.

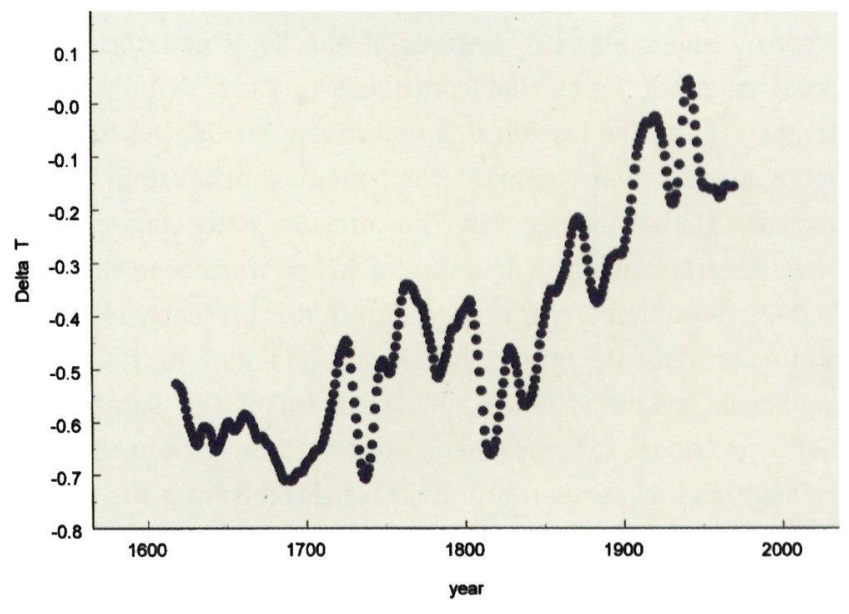

Fig. 7. Smoothed NH tropospheric temperatures since 1619, according to Moberg et al., 2005. The first nine years data are eliminated in the smoothing process. Therefore this diagram starts with the 1619 data.

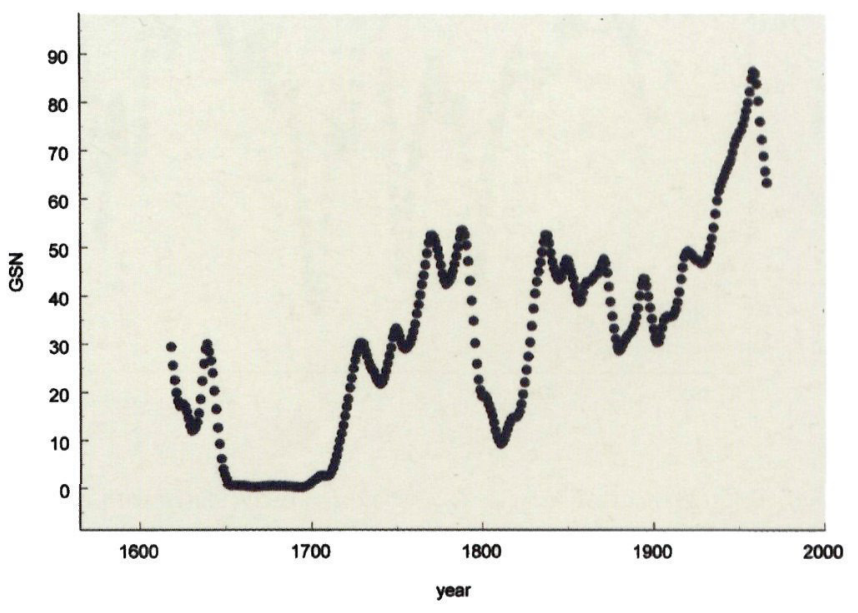

Fig. 8. Smoothed values of the sunspot number $R$ since 1619. As in Figure 7 this diagram starts with the 1619 data. 


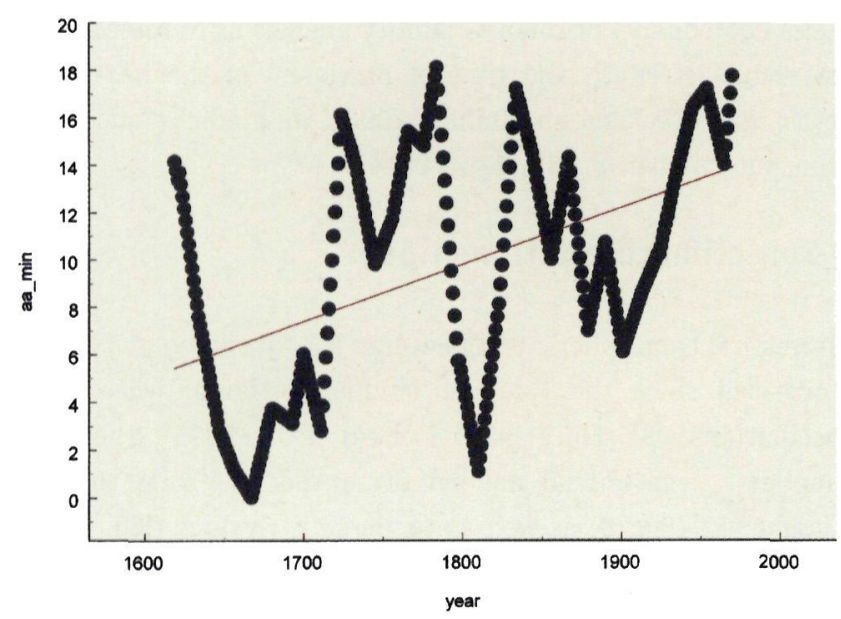

Fig. 9. Smoothed values of aamin since 1619.

This demands the least-squares solution of the equation

$$
T=y \cdot R+z \cdot a a+c \cdot \Delta t
$$

where $y$ and $z$ are the fractions of the Sun's equatorial and polar magnetic fields that contribute to Earth's tropospheric temperatures. The last term is tentatively introduced to allow for a gradual non-magnetic component contributing to the increase of the temperature. The term $\Delta t$ is the time elapsed since an arbitrary starting date, for which we choose the year 1600. In a preliminary investigation, that is presently repeated and improved, we found that about one half of the Sun's equatorial magnetic fields and one third of the Sun's polar fields contribute to tropospheric temperatures, with in addition a small gradual temperature increase. Establishing the causal solar processes of these numbers is expected to be a challenging problem.

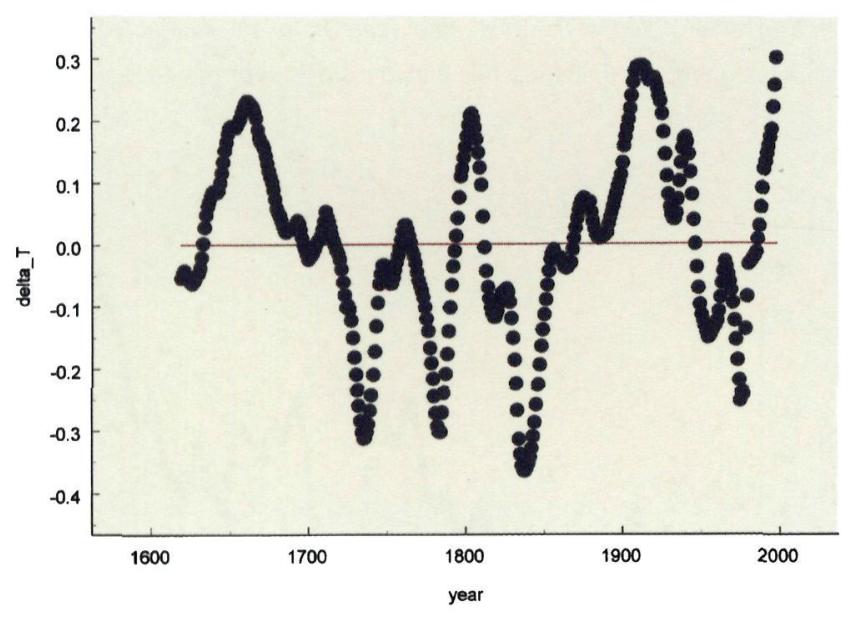

Fig. 10: Differences $\Delta T=T_{\text {obs }}-T_{\text {cal, }}$ where $T_{\text {cal }}$ is calculated with eq. (1). Peaks and troughs, relative to the calculated temperatures, alternate at a time scale of centuries.
Of significance is the fact that, in addition to the regular variations as described by eq. (1), there are, at the time scale of centuries, large temperature excursions, both positive and negative. To study them in some more detail, we plot the values over time of $T_{o b s}-T_{c a l}$, that is the difference between the observed and the calculated temperatures, where $T_{\text {cal }}$ is derived with Eq. (1). The result (Fig. 10) shows that over the past 400 years several strong temperature peaks and troughs occurred, with amplitudes of the order of $0.3^{\circ} \mathrm{C}$. Remarkable is that the recent 'global warming' peak does not seem to differ significantly from the other peaks that occurred during the last four centuries. In interpreting this diagram we should not forget that Fig. 10 does not show actual temperatures but the differences between observed and calculated solar-forced ones. The reason why the recent temperature peak appears to be so conspicuous is that it is a combination of a period of strong solar activity and a positive peak in the relative temperature. As such the recent period of global warming does not appear to be exceptional from a historical perspective.

\section{Acknowledgements}

I am obliged to an anonymous referee for very useful suggestions and to Dirk Callebaut and Silvia Duhau for our fine cooperation. For editorial suggestions I am grateful to the editor of this volume, Evert van de Graaff.

\section{References}

Akasofu, S.I. \& Frey, C.D., 1986. Heliospheric current sheet and its solar cycle variation, Journal of Geophysical Research, 91: 13679-13688.

Brun, A.S., 2004. On the interaction between differential rotation and magnetic fields in the sun, Solar Physics, 220: 333-345

Clilverd, M.A., Clarke, C., Risbeth, H., Clark, T.D.G. \& Ulich, T., 2003. Solar cycle: solar activity levels in 2100, Astronomy and Geophysics, 44, 5.20-5.21

De Jager, C., 1983. Development of the flare morphology in X-rays and the flare scenario, Solar Physics, 86: 21-30.

De Jager, C., Kuijpers, J., Correia, E. \& Kaufmann, P., 1987. A high-energy flare burst and the physical properties of its source region, Solar Physics, 110: 357-326.

De Jager, C., 2005. Solar forcing of climate; 1 . Solar variability, Space Science Reviews, 120: 197-241.

De Jager, C. \& Usoskin, I.G., 2006. On possible drivers of sun-induced climate change, Journal of Atmospheric and Solar-Terrestrial Physics, 68: 2053 .

De Jager, C. \& Duhau, S., 2008, Forecasting the parameters of sunspot cycle 24 and beyond, Journal of Atmospheric and Solar-Terrestrial Physics, submitted

Dikpati, M., De Toma, G. and Gilman, P.A., Arge, C.N. \& Withe, 0.R., 2004. Diagnosis of solar cycle 23 using a flux-transport dynamo model. Astrophysical Journal, 601: 1136.

Duhau, S., 2003. An early prediction of sunspot cycle maximum 24. Solar Physics, 213: 203. 
Duhau, S. \& Chen, C.Y., 2002. The sudden increase of solar and geomagnetic activity after 1923 as a manifestation of a non-linear solar dynamo, Geophysical Research Letters, 29, (13): 6-1.

Duhau, S. \& De Jager, C., 2008. The solar dynamo and its phase transitions during the last Millennium, Solar Physics, 250: 1-15.

Erofeev, D.V. \& Erofeeva, A.V., 2000. On the relationship between polar faculae and large-scale magnetic field, Solar Phys. 191: 281.

Feynmann, J. \& Gabriel, S.B., 1990. Period and phase of the 88-year solar cycle and the Maunder minimum - Evidence for a chaotic sun, Solar Phys. 127: 393-403.

Harvey, K.L. \& Martin, S.F., 1973. Ephemeral active regions, Solar Physics 35: 389-402.

Hundhausen, A., 1999. Coronal Mass Ejections. In: Strong, K.T., Saba, J.R.L., Haisch, B.M. \& Schmelz, J.T. (eds): 'The many phases of the Sun', Springer (New York): pp. 143-200.

Lockwood, M., Stamper, R. \& Wild, M.N., 1999. A doubling of the Sun's coronal magnetic field during the past 100 years, Nature, 399: 437-439.

Moberg, A., Sonechkin, D.M., Holmgren, K., Datsenko, N.M. \& Karlén, M., 2005. Highly variable Northern Hemisphere temperatures reconstructed from low- and high-resolution proxy data, Nature, 433: 613-617.

Muscheler, R., Beer, J. \& Kromer, B., 2003. Long-term climate variations and solar effects. In: Wu, S.T., Obridko, V., Schmieder, B. \& Sykora, J. (eds): 'TSCS Symposium, 2003', ESTEC, Noordwijk: 305-316.

Nandy, D., 2004. Exploring magnetic activity from the Sun to the stars, Solar Physics, 224: 161-169.

Ogurtsov, M.G., Nagovitsyn, J.A., Kocharov, G.E. \& Jungner, H., 2002. Longperiod cycles of the Sun's activity recorded in direct solar data and proxies, Solar Physics, 211: 371-394.

Ostriakov, V.M. \& Usoskin, I.G., 1990. On the dimension of the solar attractor, Solar Physics, 127: 405-412.

Richardson, J.G., 2004. Energetic particles and corotating interaction regions in the solar wind, Space Science Reviews, 111: 267-376.

Sakai, J.I. \& De Jager, C., 1996. Solar flares and collisions between currentcarrying loops, Space Science Reviews, 77: 1-192.

Shrivastava, P.K. \& Jaiswal, K.L., 2003. High-speed solar wind streams and cosmic-ray intensity variations during 1991-1996, Solar Physics, 214: 195-200.

Spiegel, E.A. \& Weiss, N.O., 1980. Magnetic activity and variations in solar luminosity, Nature 287: 616-619.

Stoykova, D.A., Shopov, Y.Y., Garbeva, D., Tsankov, L.T. \& Yonge, C.J., 2008. Origin of the climatic changes from orbital to sub-annual scales, Journal of Atmospheric and Solar Terrestrial Physics, 70: 293-302

Tobias, S., Hughes, D. \& Weiss, N., 2006, Unpredictable sun leaves researchers in the dark, Nature, 442: 26.

Weiss, N.O., 2002. Presidential Address, Dynamos in planets, stars and galaxies, Astronomy and Geophysics, 43: 3.9-3.13.

Weiss, N.O. \& Tobias, S.M., 2000. Physical causes of solar activity, Space Science Reviews, 94: 99-112. 